\title{
Aufgaben von Medizin und Recht: präzisierende Rechtsprechung
}

\author{
Gerhard Ebnera, Iris Herzog-Zwitter ${ }^{b}$ \\ ${ }^{a}$ Dr. med., Swiss Insurance Medicine (SIM), Präsident; ${ }^{b}$ Dr. iur., Juristin FMH Rechtsdienst, SIM Bildungsbeauftragte
}

Das Bundesgericht hat am 2. Dezember 2019 ein weiteres wegweisendes versicherungsmedizinisches Urteil gefällt, das präzisierende Anforderungen an die Begutachtung bei psychischen Erkrankungen stellt. Es präzisiert das Leiturteil BGE 141 V 281 aus dem Jahr 2015. Die Gutachterinnen und Gutachter haben sich mit der Indikatorenrechtsprechung auseinanderzusetzen und diese über weitgehend alle psychiatrischen Diagnosen hinweg gutachtlich umzusetzen.

\footnotetext{
1 BGE 130 V 352: Regeste: «Eine diagnostizierte anhaltende somatoforme Schmerzstörung allein vermag in der Regel keine lang dauernde, zu einer Invalidität führende Einschränkung der Arbeitsfähigkeit im Sinne von Art. 4 Abs. 1 IVG zu bewirken. Umschreibung der Voraussetzungen, unter welchen ein Abweichen von diesem Grundsatz ausnahmsweise in Betracht fällt.»

2 BGE 136 V 279: Regeste: «Ob eine spezifische und unfalladäquate HWS-Verletzung (Schleudertrauma) ohne organisch nachweisbare Funktionsausfälle invalidisierend wirkt, beurteilt sich sinngemäss nach der Rechtsprechung zu den anhaltenden somatoformen Schmerzstörungen (BGE $130 \mathrm{~V} 352$ E. 3).»

3 Urteil BGer 8C_753/2016 vom 15. Mai 2017 E. 4.3: «Bei leichten bis mittelschweren Störungen aus dem depressiven Formenkreis, seien sie im Auftreten rezidivierend oder episodisch, wird praxisgemäss angenommen, dass - aufgrund der nach gesicherter psychiatrischer Erfahrung regelmässig guten Therapierbarkeit-hieraus keine invalidenversicherungsrechtlich relevante Einschränkung der Arbeitsfähigkeit resultiert.»
}

Ab 2004 führten medizinisch nicht erklärbare Schmerzleiden in der Regel nicht zur Zusprache einer Invalidenrente. Man ging dann zumal von der prinzipiellen «Überwindbarkeit» der Schmerzleiden aus, wenn sie nicht gewisse Zusatzkriterien - die sogenannten «FoersterKriterien» - erfüllten. ${ }^{1}$ Diese Rechtsprechung, welche sich zunächst auf die somatoformen Schmerzstörungen bezog, wurde später auf weitere psychische Störungen nach ICD-10 (F4: Neurotische, Belastungs- und somatoforme Störungen) ausgeweitet. $^{2}$ Damit umfasste die Rechtsprechung die sogenannten "pathogenetisch-ätiologisch unklaren syndromalen Beschwerdebilder ohne nachweisbare organische Grundlage» - in die Literatur eingegangen als «PÄUSBONOG» [1].

Bei leicht und mittelgradigen depressiven Störungen galt zeitweise auch eine eigene Rechtspraxis, indem diese als prinzipiell therapierbar galten, sofern nicht von einer Therapieresistenz ausgegangen werden könne. ${ }^{3}$

Abhängigkeitsstörungen als solche führten «nach bisheriger und langjähriger höchstrichterlicher Rechtsprechung» [...] «als solche nicht zu einer rentenbegründenden Invalidität. Sie sind sozialversicherungsrechtlich erst dann relevant geworden, wenn sie eine Krankheit oder einen Unfall bewirkt haben, in deren Folge ein körperlicher oder geistiger, die Erwerbsfähigkeit beeinträchtigender Gesundheitsschaden eingetreten war, oder wenn sie selber Folge eines körperlichen oder geistigen Gesundheitsschadens waren, dem Krankheitswert zugesprochen wurde» [2].

\section{Bundesgericht ändert Rechtsprechung - Leiturteil von 2015}

Ab 2015 hat das Bundesgericht seine Rechtsprechung in Bezug auf die Beurteilung der Arbeitsfähigkeit bei psychischen Störungen im Rahmen von Verfahren im Sozialversicherungsrecht grundlegend geändert.

Im Jahre 2015 wurde nämlich vom Bundesgericht in diesem Leiturteil [3] die Überwindbarkeitsvermutung in Bezug auf somatoforme und vergleichbare psychosomatische Störungen aufgegeben und durch einen strukturierten, normativen Prüfungsraster - sogenannte Standardindikatoren - ersetzt. ${ }^{4}$ Im Jahr 2017 folgte dann die Ausweitung dieser Rechtsprechung auf depressive Störungen [4] und auf sämtliche psychische Erkrankungen [5]. Und 2019 folgte schliesslich die Ausdehnung auf die Abhängigkeitsstörungen [6].

Das letztgenannte Urteil bedeutete, dass in Bezug auf die Beurteilung der rentenbegründenden Invalidität in der Rechtsprechung die gleichen Beurteilungskriterien für alle psychischen Störungen angewendet werden. Und dies zum ersten Mal seit Einführung der Invalidenversicherung im Jahr 1960.

\section{Präzisierendes Leiturteil Ende 2019 zur Abgrenzung von Medizin und Recht}

Das Bundesgericht fällte am 2. Dezember 2019 ein weiteres, sozialversicherungsrechtliches wie versicherungsmedizinisch wegweisendes, präzisierendes Leiturteil [7]. Es befasst sich einerseits mit der Abgrenzung 
von Medizin und Recht bei den Standardindikatoren gemäss dem Leiturteil aus dem Jahr 2015 [8] und andererseits mit der Frage, wann die medizinisch-psychiatrische Folgenabschätzung aus juristischer Sicht des Rechtsanwenders Bestand hat. Mit diesem weiteren Urteil präzisiert das Bundesgericht die Aufgaben des Gutachters und attestiert der medizinischen Folgenabschätzung eine hohe Variabilität und unausweichliche Ermessenszüge.

\section{Kernaussagen aus dem Bundesgerichtsurteil}

Dieses präzisierende Leiturteil vom Dezember 2019 bestätigt im Sinne der konstanten Rechtsprechung, dass die Indikatorenrechtsprechung für die Beurteilung der Arbeitsfähigkeit bei psychischen Erkrankungen zur Anwendung kommt. Die Beurteilung der Arbeitsfähigkeit hat mit Blick auf die vorgegebenen Kriterien der Indikatorenrechtsprechung zu erfolgen, insbesondere sei der Aspekt der funktionellen Auswirkungen stärker zu berücksichtigen, was bereits in den diagnostischen Anforderungen umzusetzen sei. Unter Berücksichtigung von leistungshindernden äusseren Belastungsfaktoren wie auch von Kompensationspotenzialen (Ressourcen) sei das tatsächlich erreichbare Leistungsvermögen einzuschätzen.

Die medizinische Einschätzung des Gutachters zur Arbeitsunfähigkeit sei eine wichtige Grundlage für die Beurteilung des Rechtsanwenders, welche Arbeitsleistung der versicherten Person noch zumutbar sei. Sowohl der Mediziner als auch der Rechtsanwender hätten sich an die Vorgaben der Indikatorenrechtsprechung gemäss Leiturteil zu orientieren.

Eine freie ärztliche Arbeits(un)fähigkeitsschätzung "nach bestem Wissen und Gewissen» würde als solche den rechtlich geforderten Beweis überwiegender Wahrscheinlichkeit für das Bestehen funktioneller Einbussen und/oder verminderter Ressourcen in aller Regel nicht zu erbringen vermögen. Der Rechtsanwender könne das Ermessen des medizinisch-psychiatrischen Sachverständigen nicht zuverlässig nachvollziehen und überprüfen

Gemäss Rechtsprechung habe im Idealfall der Gutachter das Leistungsvermögen gemäss der im Leiturteil BGE 141 V 281 formulierten Fragestellungen zu beurteilen. Der Rechtsanwender hat die medizinischen Angaben dahingehend zu prüfen, ob die Indikatorenrechtsprechung bzw. der Fragenkatalog zur Anwendung gekommen ist. Der Gutachter hat die Beurteilung der Arbeitsunfähigkeit anhand der rechtserheblichen Indikatoren einzuschätzen [9]. Massgebend für den Rechtsanwender muss sein, «ob die funktionellen Auswirkun- gen medizinisch im Lichte der normativen Vorgaben widerspruchsfrei und schlüssig mit (zumindest) überwiegender Wahrscheinlichkeit nachgewiesen sind» [10]. Das Bundesgericht konstatiert, dass die ärztliche Beurteilung von der Natur der Sache her unausweichlich Ermessenszüge aufweise. Durch diese sei auch der Rechtsanwender begrenzt. Grundsätzlich könne jede Einschätzung der Arbeitsunfähigkeit durch den medizinisch-psychiatrischen Sachverständigen der (freien) Überprüfung durch die Rechtsanwender gemäss Leiturteil unterliegen. Der Rechtsanwender könne von einer medizinischen Schätzung aus triftigen Gründen abweichen, wenn die medizinisch-psychiatrische Beurteilung der Arbeitsunfähigkeit «im Ergebnis unter dem entscheidenden Gesichtswinkel betreffend Konsistenz und materieller Beweislast der versicherten, rentenansprechenden Person zu wenig gesichert ist und insofern nicht überzeugt».

\section{Die Beurteilung der Arbeitsfähigkeit hat mit Blick auf die vorgegebenen Kriterien der Indikatorenrechtsprechung zu erfolgen.}

Gemäss der Rechtsprechung bestehe «zum Einen das rechtsprechungsgemässe Verbot unzulässiger juristischer Parallelprüfung im Vergleich zur Arbeitsunfähigkeitsfestlegung durch die Gutachter». Zum anderen sieht das Leiturteil die Befugnis für den Rechtsanwender vor, im Rahmen der (freien) Überprüfung von der ärztlichen Folgenabschätzung in begründeten Fällen abzuweichen

\section{Aufgaben an die Begutachtung}

Das Bundesgericht präzisiert im vorliegenden Leiturteil [11] folgende wichtige Punkte für den Gutachter, welche in der Praxis im konkreten Fall umzusetzen sind:

- Der Gutachter hat die Arbeitsunfähigkeitseinschätzung unter Beachtung der massgebenden Indikatoren hinreichend und nachvollziehbar zu begründen.

- Der Gutachter hat substanziiert darzulegen, «aus welchen medizinisch-psychiatrischen Gründen die erhobenen Befunde das funktionelle Leistungsvermögen und die psychischen Ressourcen in qualitativer, quantitativer und zeitlicher Hinsicht zu schmälern vermögen" [12].

- Der Gutachter hat darzutun, «inwiefern und inwieweit wegen der von ihm erhobenen Befunde (Traurigkeit, Hoffnungslosigkeit, Antriebsschwäche, Müdigkeit, Konzentrations- und Aufmerksamkeitsstörungen, verminderte Anpassungsfähigkeit usw.) die beruflich-erwerbliche Arbeitsfähigkeit einge- 
schränkt ist, und zwar - zu Vergleichs-, Plausibilisierungs- und Kontrollzwecken - unter Miteinbezug der sonstigen persönlichen, familiären und sozialen Aktivitäten der rentenansprechenden Person» [13].

Sofern die medizinisch-psychiatrische Folgenabschätzung basierend auf den normierten Beweisthemen stattfindet, hat diese auch aus juristischer Sicht seitens des Rechtsanwenders Bestand [14].

\section{Fazit}

Die medizinischen Gutachterinnen und Gutachter haben sich mit der Indikatorenrechtsprechung auseinanderzusetzen und diese über weitgehend alle psychiatrischen Diagnosen hinweg gutachtlich zu beurteilen. Die Indikatoren richten sich nach versicherungsmedizinischen Grundsätzen aus, welche bereits in den entsprechenden Leitlinien zur versicherungspsychiatrischen Begutachtung ab 2016 Berücksichtigung fanden [15].

\section{Die Indikatorenrechtsprechung stellt eine wichtige Brückenfunktion zwischen Medizin und Recht dar.}

Dies bedeutet für die medizinischen Gutachter und Gutachterinnen eine hohe Verantwortung; sie haben den Fokus in der Begutachtung massgeblich auf die funktionellen Auswirkungen der jeweiligen diagnostizierten psychischen Störungen zu legen. Ferner werden sie bei der klinischen Beurteilung von Konsistenz und Plausibilität gefordert. Die gutachtliche Beurteilung der
Konsistenz ist massgeblicher Bestandteil der vorgegebenen Indikatoren; deren Ergebnis kann für die Rechtsprechung einen Ausschlussgrund darstellen [16].

Ganz neu haben sich die Gutachterinnen und Gutachter zudem mit Prognose, evidenzbasierten Behandlungsmassnahmen und funktionellen Auswirkungen von Abhängigkeitsstörungen auseinanderzusetzen, welche sie bis anhin ohne weitere Beurteilungsschritte einfach unter «Diagnosen ohne Auswirkungen auf die Arbeitsfähigkeit» aufführen konnten.

Die Indikatorenrechtsprechung stellt somit eine wichtige Brückenfunktion zwischen Medizin und Recht und damit auch eine wichtige Basis zum gegenseitigen Verständnis der beiden Wissenschaften dar.

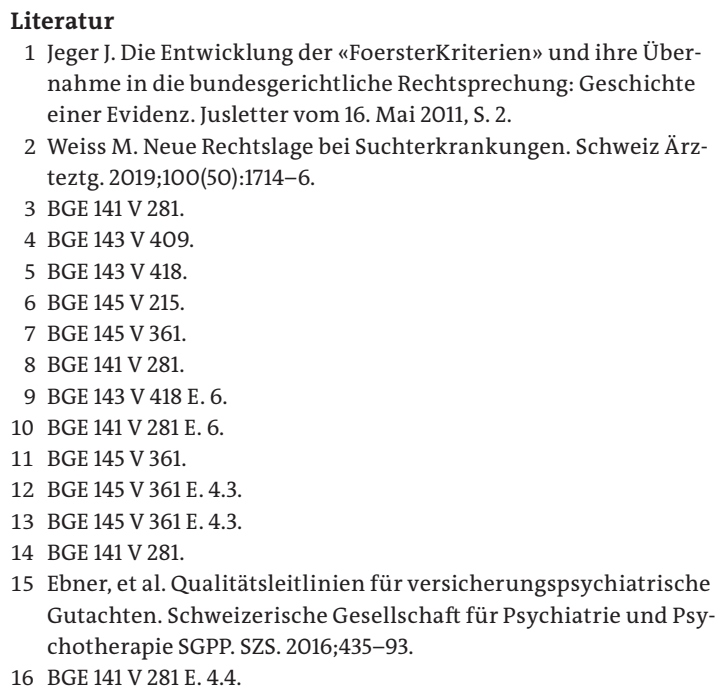

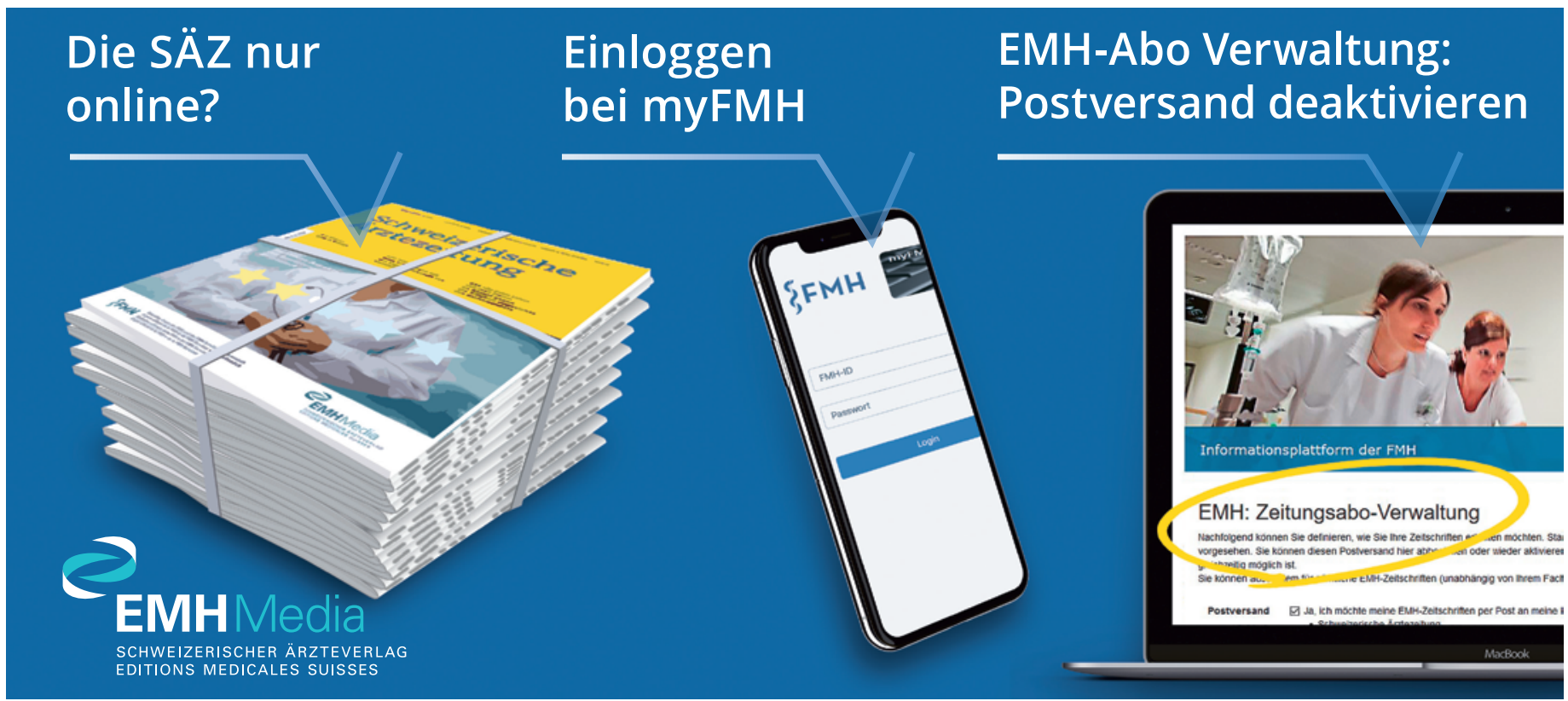

